\title{
Radiative deformation
}

\author{
V. S. Arpacia) and A. Esmaeeli \\ Department of Mechanical Engineering and Applied Mechanics, The University of Michigan, Ann Arbor, \\ Michigan 48109
}

(Received 7 September 1999; accepted for publication 13 December 1999)

\begin{abstract}
An infinitesimal change $\delta Q$ in heat flux $Q$ is shown, in terms of entropy flux $\Psi=Q / T$, to have two parts, $\delta Q=T \delta \Psi+\Psi \delta T$. The first part being the thermal displacement and the second part being the thermal deformation. Only the second part dissipates into internal energy and generates entropy. Thermodynamic arguments are extended to transport phenomena. It is shown that the thermal part of the rate of local entropy generation is related to the local rate of thermal deformation by $s^{\prime \prime \prime}=$ $-\psi_{i} / T\left(\partial T / \partial x_{i}\right)$, where $\psi_{i}=q_{i} / T, \psi_{i}$ being the rate of entropy flux vector, and $q_{i}$ the rate of heat flux vector. The part of this generation related to radiation is illustrated in terms of an example. (C) 2000 American Institute of Physics. [S0021-8979(00)06806-7]
\end{abstract}

\section{INTRODUCTION}

The concept of entropy production is now assumed well understood (see, for example, Bird, Stewart and Lightfoot, ${ }^{1}$ De Groot and Mazur ${ }^{2}$ ). Recent interest is primarily aimed at a variety of applications. Because of the size of the literature, no attempt is made here for a complete review. Some of the recent work include Arpaci and Selamet ${ }^{3}$ on the entropic efficiency of energy systems, Bejan ${ }^{4}$ on a variety of thermal problems from the view point of minimized entropy production, Lior $^{5}$ on the energy, exergy and thermomechanics of nuclear power plants, Szargut and Morris $^{6}$ on the exergy losses associated with metal production, DiVita and Brusati ${ }^{7}$ on the minimum entropy production due to ohmic dissipation in tokamaks, and $\mathrm{Kucinski}^{8}$ on the minimum entropy production in toroidal pinches. An inspection of this literature, however, reveals a need for further studies on the Second Law. Specifically, in the classical thermodynamics, the entropy difference between a system (heat reservoir at temperature $T_{1}$ ) separated by a partition from another system (heat reservoir at temperature $T_{2}$ ) is given by

$$
S_{2}-S_{1}=Q\left(\frac{1}{T_{2}}-\frac{1}{T_{1}}\right), \quad T_{1}>T_{2},
$$

$Q$ being the heat flux through the partition (Fig. 1). First note that a finite temperature drop cannot exist across a partition of no appreciable thickness. Actually, one needs to replace the ideal model of Fig. 1 dealt in thermodynamics with a real model which involves a partition of finite thickness $L$ separating two baths (Fig. 2). In this model, the bath at temperature $T_{1}$ transfers heat via an irreversible process over the thickness of partition to the bath at temperature $T_{2}$. The objective of the present study is the entropy change within the partition rather than the difference between the (internal) entropy of two baths. Toward this objective, first consider the energy balance

$$
d E=\delta Q-\delta W
$$

\footnotetext{
a)Electronic mail: arpaci@umich.edu
}

Here, $E=U+U_{K}+U_{P}$, where $U, U_{K}$, and $U_{P}$ respectively denote internal, kinetic, and potential energy, and $W=p V, p$ being the pressure and $V$ the volume. Splitting the net work,

$$
\delta W=d(p V)=V d p+p d V,
$$

and, after some regrouping, Eq. (2) becomes

$$
d U+p d V+\left[d\left(U_{K}+U_{P}\right)+V d p\right]=\delta Q .
$$

In the absence of the mechanical effects, the terms in brackets vanish and Eq. (4) is reduced to a thermal energy balance,

$$
d U+p d V=\delta Q .
$$

Now, in a manner similar to splitting work, rearranging the heat flux in terms of the entropy flux, $\Psi=Q / T$, gives

$$
\delta Q=\delta(\Psi T)=T \delta \Psi+\Psi d T .
$$

The first term on the right-hand side of Eqs. (3) and (6) respectively denotes the displaced mechanical and thermal energy, and the second term denotes the deformed mechanical and thermal energy. Only the deformed energy irreversibly dissipates into internal energy and produces entropy. Note that the pressure related volumetric change is dilation rather than deformation, it is reversible and produces no entropy, but shear stress related deformation, to be considered in the next section, is irreversible and produces entropy. Now, Eq. (5) may be rearranged in terms of Eq. (6) to give

$$
d U+p d V=T \delta \Psi+\Psi d T .
$$

Next, consider the entropy balance including a generation term, say $\Pi$, as a measure of irreversibility,

$$
d S=\delta \Psi+\delta \Pi \text {. }
$$

For the energy equivalent of Eq. (8), multiply this equation by temperature,

$$
T d S=T \delta \Psi+T \delta \Pi \text {. }
$$

Now, consider the fundamental difference,

Thermal energy balance $-T \times$ Entropy balance, obtained by subtracting Eq. (9) from Eq. (7), 


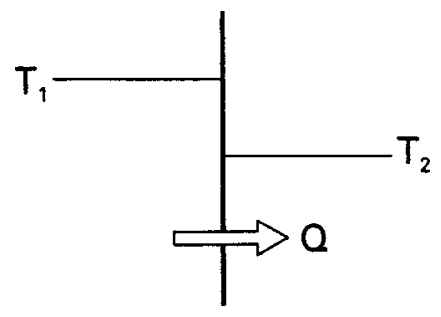

FIG. 1. Thermodynamic model—heat flux across a temperature discontinuity.

$$
d U+p d V-T d S=\Psi d T-T \delta \Pi .
$$

For a reversible process, the right-hand side of Eq. (10) vanishes and we obtain the well-known thermodynamic relation

$$
d U+p d V=T d S .
$$

For an irreversible process to be assumed locally reversible, Eq. (11) continues to be valid, and the left-hand side of Eq. (10) vanishes and the right-hand side results

$$
\delta \Pi=\frac{\Psi}{T} d T=\Psi d(\ln T)=\frac{Q}{T^{2}} d T .
$$

For a steady process within a solid partition, Eq. (5) is reduced to $d U=\delta Q=0$, and Eq. (8) to $\delta \Psi+\delta \Pi=0$. Then,

$$
\delta \Psi=-\delta \Pi=-\frac{Q}{T^{2}} d T .
$$

Now, integration of Eq. (12) between the boundaries of the partition yields

$$
\left.\Psi\right|_{1} ^{2}=-\left.\Pi\right|_{1} ^{2}=-Q \int_{1}^{2} \frac{d T}{T^{2}},
$$

or

$$
\left.\Psi\right|_{1} ^{2}=\left.Q\left(\frac{1}{T}\right)\right|_{1} ^{2}
$$

or, explicitly,

$$
\Psi_{2}-\Psi_{1}=-\left(\Pi_{2}-\Pi_{1}\right)=Q\left(\frac{1}{T_{2}}-\frac{1}{T_{1}}\right)
$$

which is identical to the right-hand side of Eq. (1) but should be identified as the the change of entropy flux across a solid partition of finite length. This change is balanced by the change of entropy production in the partition.

For a case involving both radiation and conduction, let the internal energy, heat, and work associated with gas radiation (including infrared as well as visible spectra) be $U^{R}$, $Q^{R}$, and $W^{R}$, respectively. For negligible relativistic effects,

$$
U^{R} \ll U, \quad Q^{R} \sim Q^{K}, \quad W^{R} \ll W,
$$

provided the characteristic transport velocity remains much less than the velocity of light. Then,

$$
Q=Q^{K}+Q^{R},
$$

$Q^{K}$ and $Q^{R}$ respectively being the heat flux by conduction and radiation. In the next section, the foregoing development is extended to transport phenomena.

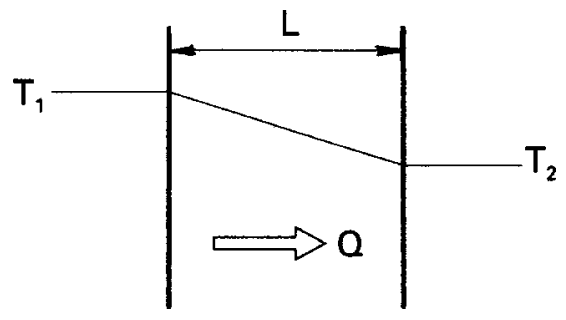

FIG. 2. Heat transfer model-heat flux over a continuous temperature difference.

The study consists of six sections: following this section which introduces thermal deformation into classical thermodynamics Section II extends the concept by incorporating the rate of thermal deformation into transport phenomena, and elaborates this deformation in terms of radiation. Section III deals with the electromagnetic deformation Section IV gives an illustrative example on entropy production resulting from these deformations Section $\mathrm{V}$ generalizes the results of Sec. IV by some dimensional arguments, and Sec. VI concludes the study.

\section{RATE OF THERMAL DEFORMATION}

Transport phenomena involve the rate of thermal deformation which requires first a consideration of the momentum balance. For a Newtonian fluid, this balance in terms of the conventional nomenclature is

$$
\rho \frac{D v_{i}}{D t}=-\frac{\partial p}{\partial x_{i}}+\frac{\partial \tau_{i j}}{\partial x_{i}}+f_{i},
$$

where $f_{i}$ includes all body forces. In terms of the rate of entropy flux, $\psi_{i}=q_{i} / T$, the local rate of entropy balance is

$$
\rho \frac{D s}{D t}=-\frac{\partial \psi_{i}}{\partial x_{i}}+s^{\prime \prime \prime},
$$

where $s^{\prime \prime \prime}$ denotes the local rate of entropy production. Also, the conservation of the rate of total energy including the rate of heat flux expressed in terms of the rate of entropy flux is

$$
\begin{aligned}
\rho \frac{D}{D t}\left(u+\frac{1}{2} v_{i} v_{i}\right)= & -\frac{\partial}{\partial x_{i}}\left(T \psi_{i}\right)-\frac{\partial}{\partial x_{i}}\left(p v_{i}\right) \\
& +\frac{\partial}{\partial x_{j}}\left(\tau_{i j} v_{i}\right)+f_{i} v_{i}+u^{\prime \prime \prime},
\end{aligned}
$$

where $u^{\prime \prime \prime}$ denotes dissipation of any energy other than thermomechanical energy into internal energy. Note that we expressed both the entropy balance and the total energy balance in terms of entropy flux $\psi_{i}$ rather than the customary approach in which the Second Law is expressed in terms of heat flux $q_{i}$. Thus, the rate of net heat flux becomes

$$
\frac{\partial q_{i}}{\partial x_{i}}=\frac{\partial}{\partial x_{i}}\left(T \psi_{i}\right)=T \frac{\partial \psi_{i}}{\partial x_{i}}+\psi_{i} \frac{\partial T}{\partial x_{i}},
$$

the first and second right-hand terms respectively denoting the rate of displaced and deformed thermal energy. One may also note that the rate of total mechanical energy associated with shear stress can be written as 


$$
\frac{\partial}{\partial x_{j}}\left(\tau_{i j} v_{i}\right)=v_{i} \frac{\partial \tau_{i j}}{\partial x_{j}}+\tau_{i j} s_{i j},
$$

where $s_{i j}$ is the rate of mechanical deformation and the first and second terms in the right-hand side denote the displaced and deformed mechanical energy, respectively. Only the deformed part of any (mechanical, thermal, electromagnetic, nuclear, chemical) energy dissipates (irreversibly converted) into internal energy. Thermal deformation in terms of entropy flux appears to be overlooked in the literature and is the motivation of this work. Now, consider the fundamental difference

$$
\begin{aligned}
& \text { Rate of total energy-(Newton's Law }{ }_{i} v_{i} \\
& -T \text { (Second Law })
\end{aligned}
$$

by subtracting the rate of mechanical energy [obtained by multiplying Eq. (14) by velocity] and the rate of thermal energy [obtained by multiplying Eq. (15) by temperature] from the rate of total energy given by Eq. (16). The result is, after some rearrangement with the conservation of mass,

$$
\frac{D \rho}{D t}+\rho \frac{\partial v_{i}}{\partial x_{i}}=0,
$$

and $\rho=1 / v$,

$$
\rho\left(\frac{D u}{D t}-T \frac{D s}{D t}+p \frac{D v}{D t}\right)=-\psi_{i} \frac{\partial T}{\partial x_{i}}+\tau_{i j} s_{i j}+u^{\prime \prime \prime}-T s^{\prime \prime \prime} .
$$

For a reversible process, all forms of deformation (that is, the right-hand side) vanish and Eq. (17) is reduced to the Gibbs thermodynamics relation

$$
\frac{D u}{D t}-T \frac{D s}{D t}+p \frac{D v}{D t}=0 .
$$

For an irreversible process to be assumed locally reversible, Eq. (18) continues to be valid and the right-hand side of Eq. (17) gives the rate of local entropy production

$$
s^{\prime \prime \prime}=\frac{1}{T}\left[-\psi_{i}\left(\frac{\partial T}{\partial x_{i}}\right)+\tau_{i j} s_{i j}+u^{\prime \prime \prime}\right],
$$

where the first and second terms in brackets respectively denotes the deformed (or dissipated) thermal and mechanical energy into internal energy, and the third term denotes any deformed (or dissipated except for thermomechanical) energy into internal energy. Next, we consider the radiative part of thermal deformation.

Thermal part of deformation may be illustrated in terms of the radiative deformation. First, consider both the conduction and radiation modes of heat transfer,

$$
q_{i}=q_{i}^{K}+q_{i}^{R} .
$$

The conductive constitution, usually described by Fourier's law,

$$
q_{i}^{K}=-k \frac{\partial T}{\partial x_{i}},
$$

is well known but the radiative constitution, depending on the assumptions made, can be described in a number of ways. Here, following Arpaci, ${ }^{9}$ consider the constitution based on the radiative stress $\Pi_{i j}$,

$$
q_{i}^{R}=-\frac{\eta}{\alpha_{M}} \frac{\partial \Pi_{i j}}{\partial x_{j}},
$$

where

$$
\Pi_{i j}=\int_{\nu} \int_{\Omega} I_{\nu} l_{i} l_{j} d \Omega d \nu,
$$

$I_{\nu}$ being the monochromatic intensity, $l_{i}$ and $l_{j}$ are spherical unit vectors, and

$$
\Pi_{i j}=\frac{4}{3} E_{b} \delta_{i j}+4 \sum_{n=1}^{\infty} \frac{1}{\alpha_{M}^{2 n}}\left(M_{i j p q} \cdot \frac{\partial}{\partial x_{p}} \frac{\partial}{\partial x_{q}}\right) E_{b}
$$

with

$$
M_{i j p q} . .=\frac{1}{4 \pi} \int_{\Omega}\left(l_{i} l_{j} l_{p} l_{q} \ldots\right) d \Omega .
$$

Here, $E_{b}=\sigma T^{4}$ is the Stefan-Boltzmann law for blackbody emissive power, $\alpha_{M}=\left(\alpha_{P} \alpha_{R}\right)^{1 / 2}$ the mean absorption coefficient, $\eta=\left(\alpha_{P} / \alpha_{R}\right)^{1 / 2}$ the degree of nongrayness, $\alpha_{P}$ and $\alpha_{R}$ respectively the Planck and Rosseland means of the absorption coefficients. $M_{i j p q}$. is an $n$ th-order moment operator. A procedure for evaluating $M_{i j p q}$. in terms of the Wallis Integrals is described in Unno and Spiege ${ }^{10}$ who apparently overlook earlier work by Milne. ${ }^{11}$ After lengthy manipulations, the procedure leads to

$$
\Pi_{i j}=4 \sum_{n=0}^{\infty} \frac{\nabla^{2 n-2}\left(2 n \partial_{i} \partial_{j}+\nabla^{2} \delta_{i j}\right) E_{b}}{\alpha_{M}^{2 n}(2 n+1)(2 n+3)},
$$

where $\partial_{i} \equiv \partial / \partial x_{i}$ and $\partial_{j} \equiv \partial / \partial x_{j}$ are used for notational convenience.

A useful alternative form of this stress explicitly involves radiative pressure, $p=(1 / 3) J$, where

$$
J=\Pi_{k k}=\sum_{n=0}^{\infty}\left(\frac{\nabla^{2}}{\alpha_{M}^{2}}\right)^{n} \frac{B}{2 n+3},
$$

$B=4 E_{b}$ and $\Pi_{k k}$ is the trace of $\Pi_{i j}$ and $J$ is the spectrally and directionally averaged monochromatic intensity,

$$
J=\int_{\nu} \int_{\Omega} I_{\nu} d \Omega d \nu
$$

For the alternative form of the stress, one follows Prandtl and Tietjens $^{12}$ in a manner similar to the development of the Stokesean viscous constitution from the Hookean elastic constitution, by rearranging Eq. (24) in terms of $\left(\frac{1}{3} J\right.$ $\left.-\Pi_{i j}\right) \delta_{i j}=0$, and obtains

$$
\Pi_{i j}=\frac{1}{3} J \delta_{i j}+\sum_{n=0}^{\infty} \frac{2 n \nabla^{2 n-2}\left[\partial_{i} \partial_{j}-(1 / 3) \nabla^{2} \delta_{i j}\right] B}{\alpha_{M}^{2 n}(2 n+1)(2 n+3)} .
$$

A first approximation for the radiative heat flux based on pressure is the well-known Eddington approximation, 


$$
q_{i}^{R}=-\frac{1}{3 \alpha_{R}} \frac{\partial J}{\partial x_{i}} .
$$

Although extensively used in the literature on gas radiation and to be utilized in the next section, the radiative flux based on this approximation deviates as much as $29 \%$ from the exact flux given by Eq. (21). In terms of this approximation, the local entropy production, resulting from the sum of thermoelectromagnetomechanical deformations, becomes

$$
s^{\prime \prime \prime}=\frac{1}{T}\left[\frac{1}{T}\left(k \frac{\partial T}{\partial x_{i}}+\frac{1}{3 \alpha_{R}} \frac{\partial J}{\partial x_{i}}\right) \frac{\partial T}{\partial x_{i}}+\tau_{i j} s_{i j}+u^{\prime \prime \prime}\right]
$$

whose radiative part based on the Eddington approximation needs to be coupled with the radiative constitution

$$
\left(\nabla^{2}-3 \alpha_{M}^{2}\right) J=-12 \alpha_{M}^{2} E_{b}
$$

(see, for example, Arpaci and Gözüm ${ }^{13}$ or Philips and Arpaci ${ }^{14}$ ). The next section illustrates local entropy production resulting from electromagnetic deformation.

\section{ELECTROMAGNETIC DEFORMATION}

For a plasma flow prescribed by MHD approximation (see, for example, Shercliff, ${ }^{15}$ Mitchner and Kruger ${ }^{16}$ ), the electromagnetic body force in Eq. (14) becomes

$$
f_{i}^{e}=\epsilon_{i j k} J_{j} B_{k}
$$

where $J_{j}$ and $B_{k}$ respectively denote the electrical current density and the magnetic field, and $J_{j}$ is given by Ohm's Law,

$$
J_{i}=\sigma\left(E_{i}+\epsilon_{i j k} v_{j} B_{k}\right),
$$

$\sigma$ being the electrical conductance and $v_{j}$ is flow velocity. For the electromagnetic power, consider the dot product of Eq. (30) with $J_{i}$,

$$
J_{i} J_{i}=\sigma\left(E_{i}+\epsilon_{i j k} v_{j} B_{k}\right) J_{i}
$$

which may be rearranged as

$$
J_{i} J_{i} / \sigma=E_{i} J_{i}+\left(\epsilon_{i j k} v_{j} B_{k}\right) J_{i}
$$

or, in terms of the identity,

$$
\left(\epsilon_{i j k} v_{j} B_{k}\right) J_{i}=-\left(\epsilon_{i j k} J_{j} B_{k}\right) v_{i},
$$

as

$$
E_{i} J_{i}=\left(\epsilon_{i j k} J_{j} B_{k}\right) v_{i}+J_{i} J_{i} / \sigma,
$$

or, in view of Eq. (29) and $u^{\prime \prime \prime}=J_{i} J_{i} / \sigma$, as

$$
E_{i} J_{i}=f_{i}^{e} v_{i}+u^{\prime \prime \prime},
$$

$E_{i} J_{i}$ being the total electromagnetic power, $f_{i}^{e} v_{i}$ and $u^{\prime \prime \prime}$ respectively the displaced and deformed (dissipated) electromagnetic power.

The foregoing considerations on the deformation energy are next illustrated in terms of an example.

\section{AN ILLUSTRATIVE EXAMPLE}

Consider a gas between two parallel plates at temperature $T_{1}$ and $T_{2}$ separated a distance $L$ apart. The emissive power and emissivity of the plates are $E_{b_{1}}$ and $E_{b_{2}}$, and $\epsilon_{1}$ and $\epsilon_{2}$, respectively. The rate of uniform energy per unit volume, $u^{\prime \prime \prime}$, is steadily generated in the gas. For negligible conduction, distribution of emissive power and entropy production between the plates are desired.

The thermal balance for a one-dimensional differential system is

$$
-d q_{x}^{R} / d x+u^{\prime \prime \prime}=0
$$

The radiative energy balance for the same system is

$$
d q_{x}^{R} / d x=\alpha_{P}\left(4 E_{b}-J\right) .
$$

Also, the one-dimensional form of Eq. (26) is

$$
q_{x}^{R}=-\left(1 / 3 \alpha_{R}\right)(d J / d x) .
$$

Now, inserting Eq. (32) into Eq. (33) gives

$$
J=4 E_{b}-u^{\prime \prime \prime} / \alpha_{P}
$$

and inserting this result into Eq. (34) yields

$$
q_{x}^{R}=-\left(4 / 3 \alpha_{R}\right)\left(d E_{b} / d x\right) .
$$

Note that Eq. (36), usually valid for a thick gas, now applies to a gas of any optical thickness because of the neglected conduction.

The combination of Eqs. (32) and (36) gives the governing equation,

$$
d^{2} E_{b} / d x^{2}+3 \alpha_{R} u^{\prime \prime \prime} / 4=0
$$

subject to the gas emissive power on the plate walls,

$$
E_{b}(0)=E_{b_{0}} \text { and } E_{b}(L)=E_{b_{L}} .
$$

Because of neglected conduction, these emissive powers are different than those imposed on the walls (the radiative jump). The solution of Eq. (37), subject to boundary conditions (38), is

$$
E_{b}(x)-E_{b_{0}}=-\left(E_{b_{0}}-E_{b_{L}}\right)(x / L)+(3 / 8) \alpha_{R} u^{\prime \prime \prime}\left(x L-x^{2}\right)
$$

which gives, in terms of Eq. (36),

$$
q_{x}^{R}=\frac{4}{3}\left(\frac{E_{b_{0}}-E_{b_{L}}}{\alpha_{R} L}\right)+\frac{u^{\prime \prime \prime}}{2}(2 x-L) .
$$

However, Eqs. (39) and (40) need to be expressed in terms of the wall emissive powers rather than wall values of the gas emissive power. To accomplish this, consider the radiative boundary conditions (see, e.g., Goody ${ }^{17}$ or Arpaci and $\operatorname{Troy}^{18}$ ),

$$
E_{b_{1}}-E_{b_{0}}=\left(\frac{1}{\epsilon_{1}}-\frac{1}{2}\right) q_{x}^{R}(0)-\frac{1}{4 \alpha_{P}} \frac{d q_{x}^{R}(0)}{d x},
$$

and

$$
E_{b_{2}}-E_{b_{L}}=\left(\frac{1}{\epsilon_{2}}-\frac{1}{2}\right) q_{x}^{R}(L)-\frac{1}{4 \alpha_{P}} \frac{d q_{x}^{R}(L)}{d x},
$$

which may be rearranged in terms of Eq. (32) to give

$$
E_{b_{1}}-E_{b_{0}}=\left(\frac{1}{\epsilon_{1}}-\frac{1}{2}\right) q_{x}^{R}(0)-\frac{u^{\prime \prime \prime}}{4 \alpha_{P}}
$$

and 


$$
E_{b_{2}}-E_{b_{L}}=\left(\frac{1}{\epsilon_{2}}-\frac{1}{2}\right) q_{x}^{R}(L)-\frac{u^{\prime \prime \prime}}{4 \alpha_{P}} .
$$

The difference between Eqs. (43) and (44) yields

$$
\begin{aligned}
E_{b_{1}}-E_{b_{2}}= & \left(E_{b_{0}}-E_{b_{L}}\right)+\left(\frac{1}{\epsilon_{1}}-\frac{1}{2}\right) q_{x}^{R}(0) \\
& +\left(\frac{1}{\epsilon_{2}}-\frac{1}{2}\right) q_{x}^{R}(L),
\end{aligned}
$$

where, employing Eq. (40),

$$
q_{x}^{R}(0)=\frac{4}{3}\left(\frac{E_{b_{0}}-E_{b_{L}}}{\alpha_{R} L}\right)-\frac{u^{\prime \prime \prime} L}{2}
$$

and

$$
q_{x}^{R}(L)=\frac{4}{3}\left(\frac{E_{b_{0}}-E_{b_{L}}}{\alpha_{R} L}\right)+\frac{u^{\prime \prime \prime} L}{2} .
$$

Note that

$$
-q_{x}^{R}(0)+q_{x}^{R}(L)=u^{\prime \prime \prime} L,
$$

as expected. Now, from Eqs. (45), (46), and (47), after some manipulations,

$$
E_{b_{0}}-E_{b_{L}}=\frac{\left(E_{b_{1}}-E_{b_{2}}\right)+\frac{u^{\prime \prime \prime} L}{2}\left(\frac{1}{\epsilon_{1}}-\frac{1}{\epsilon_{2}}\right)}{1+\frac{4 \eta}{3 \tau_{M}}\left(\frac{1}{\epsilon_{1}}+\frac{1}{\epsilon_{2}}-1\right)},
$$

$\tau_{M}=\alpha_{M} L$ being the optical thickness. Then, $q_{x}^{R}$, in terms of the imposed wall emissive powers, obtained by inserting Eq. (49) into Eq. (40), is

$$
q_{x}^{R}=\frac{\left(E_{b_{1}}-E_{b_{2}}\right)+\frac{u^{\prime \prime \prime} L}{2}\left(\frac{1}{\epsilon_{1}}-\frac{1}{\epsilon_{2}}\right)}{\frac{3 \tau_{M}}{4 \eta}+\frac{1}{\epsilon_{1}}+\frac{1}{\epsilon_{2}}-1}+\frac{u^{\prime \prime \prime}}{2}(2 x-L) .
$$

Also, for $x=0$,

$$
q_{x}^{R}(0)=\frac{\left(E_{b_{1}}-E_{b_{2}}\right)+\frac{u^{\prime \prime \prime} L}{2}\left(\frac{1}{\epsilon_{1}}-\frac{1}{\epsilon_{2}}\right)}{\frac{3 \tau_{M}}{4 \eta}+\frac{1}{\epsilon_{1}}+\frac{1}{\epsilon_{2}}-1}-\frac{u^{\prime \prime \prime} L}{2} .
$$

For $u^{\prime \prime \prime}=0$ and $\tau_{M}=0$, Eq. (51) is reduced to the enclosure radiation between two closely located parallel large plates, as expected.

To express $E_{b}$ of Eq. (39) in terms of the wall emissive powers, rearrange Eq. (43) as

$$
E_{b_{0}}=E_{b_{1}}-\left(\frac{1}{\epsilon_{1}}-\frac{1}{2}\right) q_{x}^{R}(0)+\frac{u^{\prime \prime \prime}}{4 \alpha_{P}} .
$$

Then, in terms of Eqs. (49) and (52),

$$
\begin{aligned}
E_{b}(x)-E_{b_{1}}= & -\left(\frac{1}{\epsilon_{1}}-\frac{1}{2}+\frac{3 \tau_{M} x}{4 \eta L}\right) q_{x}^{R}(0) \\
& +\frac{u^{\prime \prime \prime} L}{4 \eta}\left(\frac{1}{\tau_{M}}-\frac{3 \tau_{M} x^{2}}{2 L^{2}}\right),
\end{aligned}
$$

where $q_{x}^{R}(0)$ is given by Eq. (51).

To evaluate the radiative entropy production, consider the one-dimensional thermal part of Eq. (19) in terms of the radiative heat flux,

$$
s^{\prime \prime \prime}=-\frac{q_{x}^{R}}{T^{2}}\left(\frac{d T}{d x}\right)
$$

and replace $q_{x}^{R}$ in the above equation in terms of Eq. (36). Thus,

$$
s^{\prime \prime \prime}=\frac{4}{3 \alpha_{R} T^{2}}\left(\frac{d E_{b}}{d x}\right)\left(\frac{d T}{d x}\right) .
$$

Next, substitute for $d T / d x$ and $d E_{b} / d x$ in the above using Eq. (53) and noting that $E_{b}=\sigma T^{4}$ to get

$$
s^{\prime \prime \prime}=\left(\frac{3 L}{16 \tau \sigma T^{5}}\right)\left(\frac{\tau_{M}}{\eta L}\right)^{2}\left(q_{x}^{R}(0)+u^{\prime \prime \prime} x\right)^{2} .
$$

This entropy production is now evaluated for the two special cases that follow.

\section{A. Stagnant gas between two plates}

In this case, $u^{\prime \prime \prime}=0$, and heat flux is constant [i.e., $\left.q_{x}^{R}(0)=q_{x}^{R}(L)=q_{x}^{R}(x)\right]$. Thus, Eq. (55) reduces to

$$
s^{\prime \prime \prime}=\left(\frac{3 L}{16 \tau \sigma T^{5}}\right)\left(\frac{\tau_{M}}{\eta L}\right)^{2}\left[q_{x}^{R}(0)\right]^{2},
$$

or

$$
4 \sigma T^{5} L s^{\prime \prime \prime}=\left(\frac{3 \tau}{4}\right)\left(q_{x}^{R}\right)^{2} .
$$

For this special case, Eq. (45) reduces to

$$
E_{b_{1}}-E_{b_{2}}=\left(E_{b_{0}}-E_{b_{L}}\right)+\left(\frac{1}{\epsilon_{1}}+\frac{1}{\epsilon_{2}}-1\right) q_{x}^{R},
$$

which in terms of Eqs. (46) or (47), gives

$$
q_{x}^{R}=\frac{E_{b_{1}}-E_{b_{2}}}{3 \tau / 4+1 / \epsilon_{1}+1 / \epsilon_{2}-1} .
$$

Substitution of the above relation in Eq. (56) results in

$$
\frac{4 \sigma T^{5} s^{\prime \prime \prime} L / E_{b_{1}}^{2}}{\left(1-E_{b_{2}} / E_{b_{1}}\right)^{2}}=\frac{3 \tau / 4}{\left(3 \tau / 4+1 / \epsilon_{1}+1 / \epsilon_{2}-1\right)^{2}} .
$$

Figure 3 shows the radiative entropy production versus optical thickness $\tau$ for the special cases of $\epsilon_{1}=\epsilon_{2}=\epsilon$. As expected, this production diminish for $\tau=0$ and $\tau \rightarrow \infty$, and it goes through a maximum at an intermediate thickness,

$$
\tau=\frac{4}{3}\left(\frac{1}{\epsilon_{1}}+\frac{1}{\epsilon_{2}}-1\right),
$$

which, for black surfaces, becomes $\tau=4 / 3$. An inspection of Eq. (57) reveals that

$$
s^{\prime \prime \prime} T^{5} L=f\left(\tau, E_{b_{1}}, E_{b_{2}}, \epsilon_{1}, \epsilon_{2}\right) .
$$

Then, for specified temperature, emissivities, gas optical thickness, and geometry, 


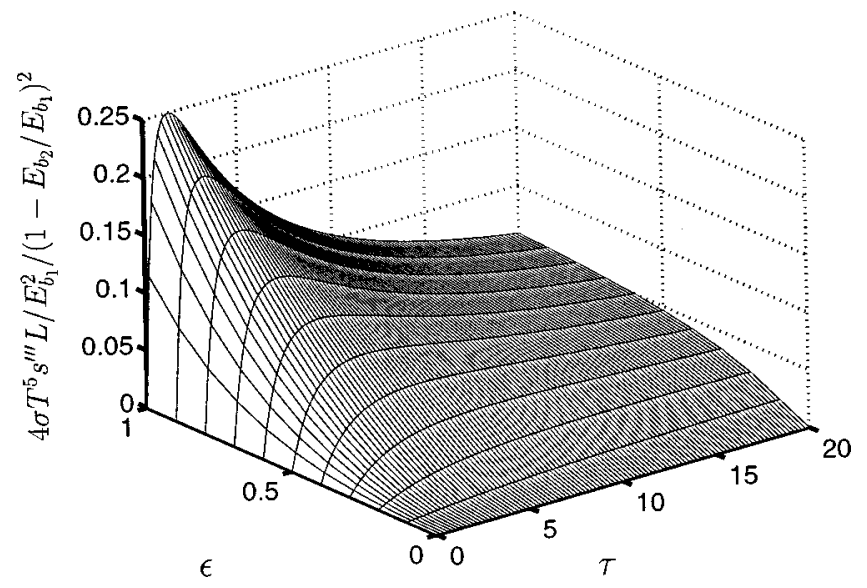

FIG. 3. Entropy production vs optical thickness and emissivity in a stagnant gas between two plates.

$$
s^{\prime \prime \prime} T^{5}=\text { Const. }
$$

which can also be seen directly from Eq. (57).

\section{B. Plasma between two plates}

Let the gas be an electrically conducting fluid. In a fully developed plasma flow (say, for example, the Hartman flow), the Joulean dissipation is spacewise distributed. Here, only for mathematical convenience, we assume $u^{\prime \prime \prime}$ to be uniform. For $T_{1}=T_{2}=T_{w}\left(E_{b_{1}}=E_{b_{2}}=E_{b_{w}}\right)$ and $\epsilon_{1}=\epsilon_{2}=\epsilon_{w}$, Eqs. (39) and (40) yield, in terms of Eqs. (45), (46), (48), and considering the fact that now $q_{x}^{R}=\left(u^{\prime \prime \prime} / 2\right)(2 x-L)$ and $q_{x}^{R}(0)=-u^{\prime \prime \prime} L / 2$,

$$
E_{b}(x)-E_{b w}=\frac{u^{\prime \prime \prime} L}{2}\left[\frac{1}{\epsilon_{w}}-\frac{1}{2}+\frac{1}{2 \eta \tau_{M}}+\frac{3 \tau_{M} x}{4 \eta L}\left(1-\frac{x}{L}\right)\right] .
$$

Then, Eq. (55) gives in terms of Eq. (58)

$$
\begin{aligned}
& \frac{s^{\prime \prime \prime}}{u^{\prime \prime \prime} T_{w}} \\
& =\frac{\frac{3 \tau_{M}}{8 \eta}\left(\frac{u^{\prime \prime \prime} L}{2 E_{b_{w}}}\right)\left(\frac{1}{2}-\frac{x}{L}\right)^{2}}{\left\{1+\left(\frac{u^{\prime \prime \prime} L}{2 E_{b_{w}}}\right)\left[\frac{1}{\epsilon_{w}}-\frac{1}{2}+\frac{1}{2 \eta \tau_{M}}+\frac{3 \tau_{M} x}{4 \eta L}\left(1-\frac{x}{L}\right)\right]\right\}^{5 / 4} .} .
\end{aligned}
$$

Figure 4 shows (for $\eta=1, \epsilon_{w}=1$, and $u^{\prime \prime \prime} L / 2 E_{b_{w}}=1$ ) the distribution of the entropy production versus the optical thickness for two locations. Optical behavior of the foregoing two cases is now generalized by an order of magnitude analysis.

\section{DIMENSIONAL ARGUMENTS}

Consider the thermal part of Eq. (19),

$$
s^{\prime \prime \prime}=\frac{1}{T}\left[\frac{1}{T}\left(k \frac{\partial T}{\partial x_{i}}+\frac{1}{3 \alpha_{R}} \frac{\partial J}{\partial x_{i}}\right)\left(\frac{\partial T}{\partial x_{i}}\right)\right] .
$$

Introduce an entropy production number,

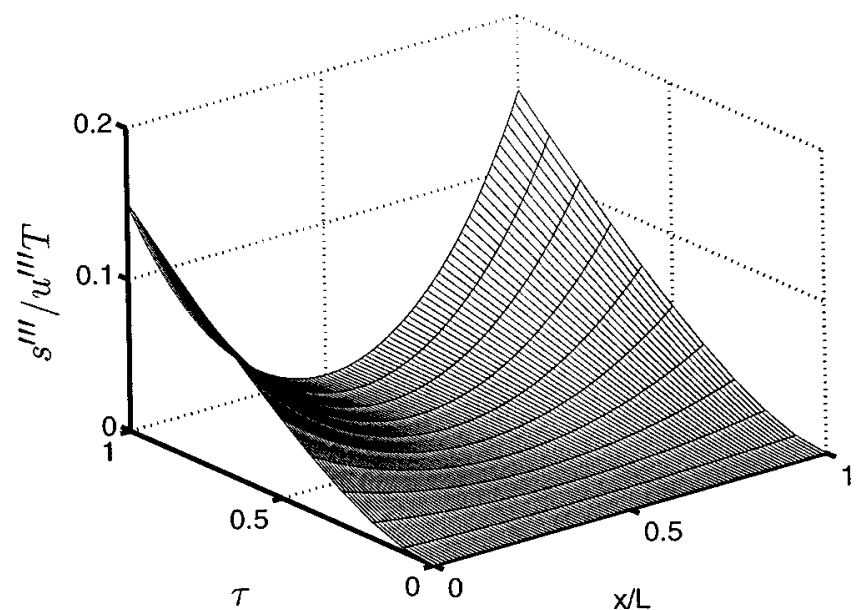

FIG. 4. Entropy production vs optical thickness and location in a plasma between two plates.

$$
\Pi_{s}=s^{\prime \prime \prime} l^{2} / k,
$$

$l$ being a characteristic length, and a heat transfer number,

$$
H=\frac{\left(\partial J / \partial x_{i}\right) / 3 \alpha_{R}}{k\left(\partial T / \partial x_{i}\right)}=\frac{q_{i}^{R}}{q_{i}^{K}} .
$$

In terms of these numbers, Eq. (60) becomes

$$
\Pi_{s}=(1+H) \frac{l^{2}}{T^{2}}\left(\frac{\partial T}{\partial x_{i}}\right)\left(\frac{\partial T}{\partial x_{i}}\right) .
$$

Also, from Eq. (26), on dimensional grounds,

$$
q^{R} \sim\left(J_{w}-J_{\infty}\right) / 3 \alpha_{R} \delta,
$$

where $\delta$ is the thickness of thermal boundary layer and $J_{w}$ and $J_{\infty}$ are the wall and ambient values of $J$, respectively. To relate $J$ to temperature, consider the radiative constitution given by Eq. (28). Using Fourier transforms, for example, $\exp \left(i k_{j} x_{j}\right), k_{j}$ being the wave number vector,

$$
\nabla^{2}=-k_{0}^{2}, \quad k_{0}^{2}=k_{1}^{2}+k_{2}^{2}+k_{3}^{2}
$$

or, in view of $k_{0} \sim \delta^{-1}, \nabla^{2} \sim-\delta^{2}$, and Eq. (28) yields

$$
\left(\delta^{-2}+3 \alpha_{M}^{2}\right) J \sim 12 \alpha_{M}^{2} E_{b} .
$$

Then, in terms of the optical thickness,

$$
\begin{aligned}
& \tau \sim \alpha_{M} \delta, \\
& J \sim\left(\frac{12 \tau^{2}}{1+3 \tau^{2}}\right) E_{b},
\end{aligned}
$$

which, together with Eq. (64), leads to the radiative heat flux,

$$
q^{R} \sim 4 \eta\left[\tau /\left(1+3 \tau^{2}\right)\right]\left(E_{b_{w}}-E_{b_{\infty}}\right),
$$

which is valid for any optical thickness but excludes any boundary effect. For $\eta=1$, Fig. 5 shows the radiative heat flux versus emission and absorption, which, respectively, are the measures for the hotness and thickness of the gas.

To include any wall effect in Eq. (68), first consider the boundary-affected thick gas and thin gas approximations with negligible scattering. For the thick gas, from Arpaci and Larsen $^{19}$ 


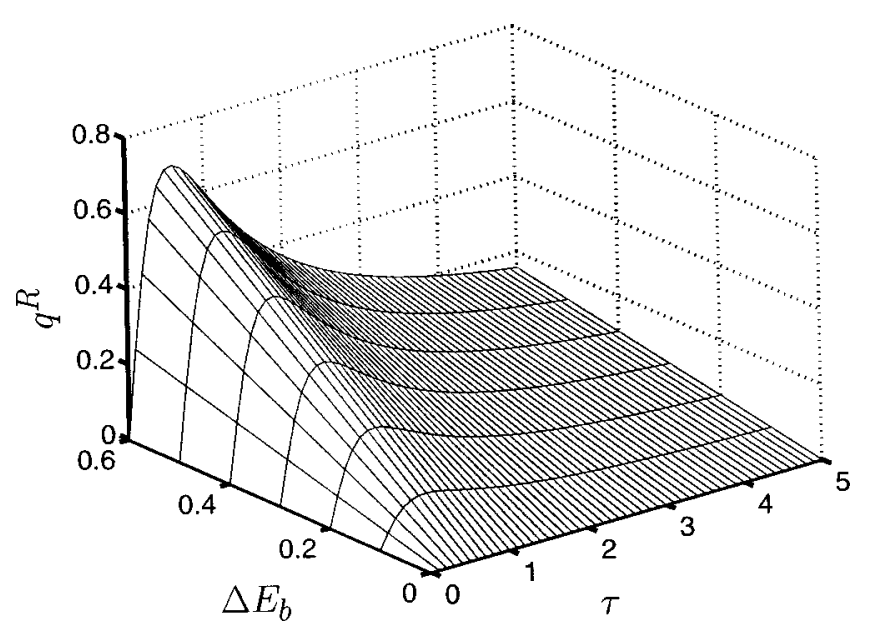

FIG. 5. Radiative heat flux vs emission and absorption.

$$
q_{y}^{R}=\frac{-4}{3 \alpha_{R}}\left(1-\rho_{w} E_{3}-\frac{3}{2} E_{4}\right) \frac{\partial E_{b}}{\partial y},
$$

where $\rho_{w}$ is the wall reflectivity and $E_{3}$ and $E_{4}$ are the usual exponential integrals of order three and four. On boundaries,

$$
\left.q_{y}^{R}\right|_{w}=\left.\frac{-4}{3 \alpha_{R}}\left(\frac{\epsilon_{w}}{2}\right) \frac{\partial E_{b}}{\partial y}\right|_{w} .
$$

However, near a boundary, the Rosseland gas gives

$$
q_{y}^{R}=-\frac{4}{3 \alpha_{R}} \frac{\partial E_{b}}{\partial y}
$$

and the net radiative source, obtained from the difference between Eqs. (70) and (71), is

$$
\frac{\partial q_{y}^{R}}{\partial y}=\frac{4}{3 \alpha_{R}}\left(1-\frac{\epsilon_{w}}{2}\right) \frac{\partial E_{b}}{\partial y}
$$

whose integration yields, on dimensional grounds,

$$
q_{w}^{R}=\frac{4 \eta}{3 \tau}\left(1-\frac{\epsilon_{w}}{2}\right)\left(E_{b_{w}}-E_{\infty}\right),
$$

where $\epsilon_{w}$ is the wall emissivity.

For the thin gas, Arpaci and Troy ${ }^{18}$ show

$$
\partial q_{y}^{R} / \partial y=4 \alpha_{P}\left[\left(E_{b}-E_{b_{\infty}}\right)-\frac{\epsilon_{w}}{2}\left(E_{b_{w}}-E_{b_{\infty}}\right) E_{2}\right],
$$

where $E_{2}$ is the exponential integral of order two. On boundaries,

$$
\left.\frac{\partial q_{y}^{R}}{\partial y}\right|_{w}=4 \alpha_{P}\left(1-\frac{\epsilon_{w}}{2}\right)\left(E_{b_{w}}-E_{b_{\infty}}\right)
$$

whose integration yields, on dimensional grounds,

$$
q_{w}^{R} \sim 4 \eta \tau\left(1-\frac{\epsilon_{w}}{2}\right)\left(E_{b_{w}}-E_{b_{\infty}}\right) .
$$

Thus, the radiative heat flux, including a hot wall as well as the emission and absorption effects, is found to be

$$
q^{R} \sim 4 \eta\left(1-\frac{\epsilon_{w}}{2}\right)\left(\frac{\tau}{1+3 \tau^{2}}\right)\left(E_{b_{w}}-E_{b_{\infty}}\right) .
$$

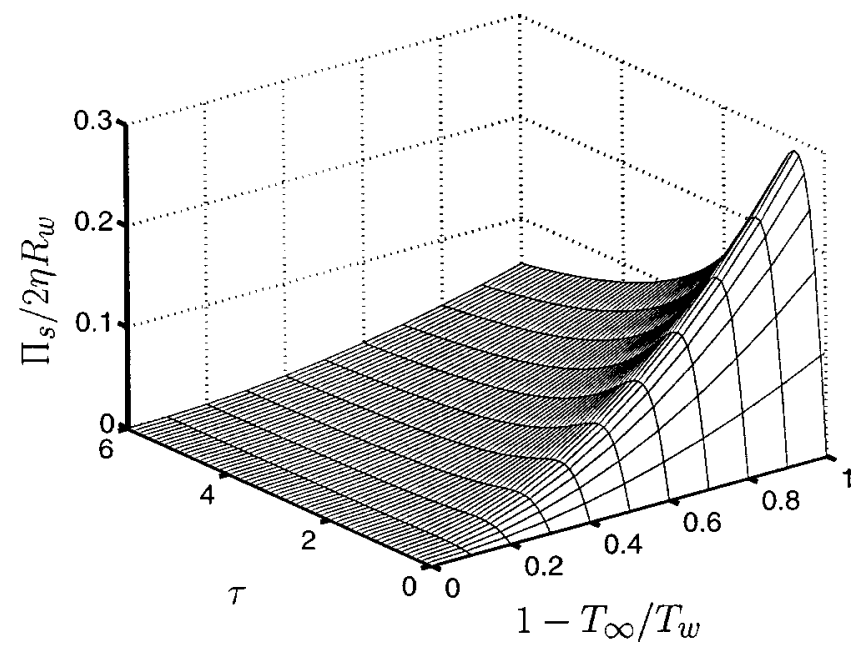

FIG. 6. Radiative entropy production vs temperature difference and optical thickness.

Furthermore, introducing a dimensionless number,

$$
\mathscr{B}_{w}=\frac{\text { Emission }}{\text { Conduction }} \sim \frac{E_{b_{w}}-E_{b_{\infty}}}{k\left(T_{w}-T_{\infty}\right) / \delta},
$$

and Eq. (62) may be rearranged as

$$
H_{w}=\frac{q_{w}^{R}}{q_{w}^{K}} \sim 4 \eta\left(1-\frac{\epsilon_{w}}{2}\right)\left(\frac{\tau}{1+3 \tau^{2}}\right) \mathscr{R}_{w} .
$$

Finally, Eq. (63) yields, in terms of Eq. (79),

$$
\Pi_{s} \sim\left[\left(T_{w}-T_{\infty}\right) / T\right]^{2}\left(1+H_{w}\right),
$$

or, explicitly,

$$
\Pi_{s} \sim\left(\frac{T_{w}-T_{\infty}}{T}\right)^{2}\left[1+4 \eta\left(1-\frac{\epsilon_{w}}{2}\right)\left(\frac{\tau}{1+3 \tau^{2}}\right) \mathscr{B}_{w}\right] .
$$

The smallest value of this production is on the hot boundary, and its radiative part becomes, after some rearrangement,

$$
\frac{\Pi_{s}}{4 \eta\left(1-\epsilon_{w} / 2\right) \mathscr{B}_{w}} \sim\left(1-\frac{T_{\infty}}{T_{w}}\right)^{2}\left(\frac{\tau}{1+3 \tau^{2}}\right) .
$$

For a proportionality constant of unity [chosen arbitrarily for a graphical representation of Eq. (82) and $\left.\epsilon_{w}=1\right]$, Fig. 6 shows the boundary production of radiative entropy versus the optical thickness and the temperature ratio.

\section{CONCLUSIONS}

The present study is based on the original idea suggesting a First Law in terms of entropy flux rather than the wellknown classical approach expressing the Second Law in terms of heat flux. Accordingly, heat flux is expressed by a product of temperature and entropy flux. A change in heat flux is identified as a combination of thermal displacement and thermal deformation. Only thermal deformation dissipates (irreversibly transforms) into internal energy and produces entropy. Using the concept of thermal deformation, the well-known thermodynamic irreversibility across a thermal discontinuity is replaced by actual irreversibility across a continuous thermal distribution. These considerations allow 
a transition from ideal concepts introduced in classical thermodynamics to actual concepts utilized in transport phenomena. The entropy production resulting from the thermal deformation is illustrated in terms of a radiative example.

${ }^{1}$ R. B. Bird, W. E. Stewart, and E. N. Lightfoot, Transport Phenomena (Wiley, New York, 1960).

${ }^{2}$ S. R. De Groot and P. Mazur, Non-equilibrium Thermodynamics (NorthHolland, Amsterdam, 1962).

${ }^{3}$ V. S. Arpaci and A. Selamet, Prog. Energy Combust. Sci. 19, 429 (1992).

${ }^{4}$ A. Bejan, J. Appl. Phys. 79, 1191 (1996).

${ }^{5}$ N. Lior, Energy Convers. Manage. 38, 1585 (1997).

${ }^{6}$ J. Szargut and D. R. Morris, Int. J. Energy Res. 14, 605 (1990).

${ }^{7}$ A. DiVita and M. Brusati, Plasma Phys. Controlled Fusion 37, 1075 (1995).

${ }^{8}$ M. Y. Kucinski, Plasma Phys. Controlled Fusion 40, 111 (1998).
${ }^{9}$ V. S. Arpaci, Microscales of Turbulence (Gordon and Breach, New York, 1997).

${ }^{10}$ W. Unno and E. A. Spiegel, Publ. Astron. Soc. Jpn. 18, 85 (1966).

${ }^{11}$ E. A. Milne, Handbuch der Astrophysik 3, 65 (1930).

${ }^{12}$ L. Prandtl and O. G. Tietjens, Fundamentals of Hydro- and Aeromechanics (Dover, New York, 1957).

${ }^{13}$ V. S. Arpaci and D. Gözüm, Phys. Fluids 16, 581 (1973).

${ }^{14}$ W. F. Philips and V. S. Arpaci, J. Plasma Phys. 13, 523 (1975).

${ }^{15}$ J. A. Shercliff, A Textbook of Magnetohydrodynamics (Pergamon, Oxford, 1965).

${ }^{16}$ M. Mitchner and C. H. Kruger, Partially Ionized Gases (Wiley, New York, 1973)

${ }^{17}$ R. M. Goody, Atmospheric Radiation (Oxford University Press, London, 1964).

${ }^{18}$ V. S. Arpaci and S. Troy, J. Thermophys. Heat Transfer 4, 407 (1990).

${ }^{19}$ V. S. Arpaci and P. S. Larsen, AIAA J. 7, 602 (1969). 